\title{
TTR
}

Traduction, terminologie, re?daction

\section{Barbara Folkart. Le Conflit des énonciations. Traduction et discours rapporté. Montréal, Les Éditions Balzac, 1991, 481 p.}

\section{Agnès Whitfield}

Volume 5, numéro 2, 2e semestre 1992

Kafka pluriel : réécriture et traduction

URI : https://id.erudit.org/iderudit/014746ar

DOI : https://doi.org/10.7202/014746ar

Aller au sommaire du numéro

Éditeur(s)

Association canadienne de traductologie

ISSN

0835-8443 (imprimé)

1708-2188 (numérique)

Découvrir la revue

Citer ce compte rendu

Whitfield, A. (1992). Compte rendu de [Barbara Folkart. Le Conflit des

énonciations. Traduction et discours rapporté. Montréal, Les Éditions Balzac, 1991, 481 p.] TTR, 5(2), 257-260. https://doi.org/10.7202/014746ar d'utilisation que vous pouvez consulter en ligne.

https://apropos.erudit.org/fr/usagers/politique-dutilisation/ 
and failings, set against a wide variety of perspectives, from literature to theology." (p. 150)

Finally, a last paper, "Problems of Editing a Translation: Anglo-Norman to Middle English," by C.W. MARX, raises the problems involved in editing a translation by lack of access to the source exemplar.

As will be gathered from this very rapid presentation of the papers contained in these two volumes, a wealth of material is to be found therein, exemplifying for the most part nevertheless certain basic recurrent themes which seem to be an essential aspect of any reflection on translation. There is of course a need always to consider such themes within the particular context in which they occur, and indeed, the obligation to contextualise is a question these papers raise. Such a collection, in its very diversity, makes it possible for the reader to get a sense of the multiplicity of practices and approaches which existed in medieval times, and for this alone it is extremely useful. Other themes as well - the limits of translation (its relation to rewriting, to adaptation and to imitation), the role of translation in terms of national literatures and languages, and the relation of translators to their readers/audience - are also still of importance to us. Thus the interest of the papers presented here for anyone concerned with the history and theory of translation.

Paul St-Pierre

Université Laval

\section{Barbara FOLKART. Le Conflit des énonciations. Tra- duction et discours rapporté. Montréal, Les Éditions Balzac, 1991, 481 p.}

L'Altérité essentielle de la traduction: le faire producteur du sujet traduisant

C'est contre une certaine conception de la traduction traversée encore par le mythe de la fidélité et par la nostalgie de la transparence que Barbara Folkart s'élève dans cet ouvrage aux prolongements nombreux tant pour la pragmatique et la théorie littéraire que pour la traduction. Car, plutôt que 
de considérer la traduction comme une simple réplique de l'original, un texte d'où peuvent, et doivent, $s$ 'effacer tout le travail de ré-énonciation, tous ces infra-phénomènes coriaces qui résistent à l'équivalence, elle accepte d'emblée l'altérité inévitable du texte traduit, cette «altérité essentielle qu'il faut, non pas refouler, mais assumer, en mettant en évidence les déviances mêmes minimes qui la fondent» (p. 13). S'inspirant de l'intuition de Brian Mossop qui envisage le traducteur comme un «rapporteur», elle formule ainsi un modèle de la traduction qui situe celle-ci dans le contexte d'un "vouloir-redire», en choisissant comme point de départ de l'analyse, non pas le texte original, mais l'activité du sujet traduisant, son insertion dans le processus de ré-énonciation.

Le premier chapitre de l'ouvrage est consacré à la mise en place d'un outillage conceptuel. L'auteure analyse quelques approches théoriques de l'énonciaton (entre autres, celles élaborées par Benveniste et Récanati), en vue de formuler un modèle complexe, mais fonctionnel, apte à tenir compte des nombreux enjeux de la ré-énonciation, qu'elle soit interlinguale ou intralinguale. Sa sensibilité, en tant que traductologue, à la verbalité de l'énoncé, et à ses implications pour la portée de celui-ci, l'amène à nuancer le schème bidimensionel avancé par Récanati. Aussi insiste-t-elle non seulement sur «la solidarité qui existe entre marge et proposition» (p. 20), mais encore sur le fait que l'énonciation est «construction d'une sémiotique discursive en même temps que mise en place d'un repérage [d'un contexte pragmatique] et d'une référence [d'un contenu propositionnel]» (p. 31). Ce schème tridimensionnel lui permet ensuite de mieux cerner les différentes distorsions opérées par la citation, toujours dans le but de voir dans quelle mesure on peut rapprocher le fonctionnement des discours rapportés au processus de traduction en tant que ré-énonciation. Tant par l'analyse que par la typologie des divers filtrages ou processus de re-médiation du discours direct ou indirect qu'elle propose, cette partie de l'ouvrage intéresserait non seulement les traductologues, mais tout spécialiste de théorie littéraire préoccupé par la question de la citation et de certains phénomènes d'intertextualité.

A la fois mise en application et prolongement du chapitre initial, le deuxième chapitre du livre explore la question de la traduction de discours rapportés, alors que la traduction, envisagée elle-même comme une sorte de discours rapporté intralingual, doit intervenir à son tour sur des discours déjà cités dans un contexte interlingual. De nouveau, un des grands mérites du travail de Barbara Folkart consiste à fournir une analyse systématique de toute une gamme de phénomènes variés qui, d'une manière 
ou d'une autre, reflètent un certain gauchissement ré-énonciatif: la citation intertextuelle, la citation affichée, la citation phagocytée, la sollicitation du fragment cité, la re-sémantisation du fragment cité, la citation hybride, la citation par rupture discursive. Partant d'exemples probants, tirés pour la plupart de traductions littéraires, elle montre que, «comme le discours rapporté, la traduction n'est jamais une transformation nulle»:

Elle ajoute toujours de la valeur, crée inévitablement des distorsions (bruit), des décalages ou des sollicitations (modulation) qui permettent de toucher du doigt à la ré-énonciation, de mesurer le décalage énonciatif qui se creuse entre la voix de l'instance de réénonciation et celle de l'instance d'énonciation, d'appréhender le dire du traducteur en flagrant délit de conflit avec celui de l'auteur. (p. 127)

Nous voici donc au cœur de ce «conflit des énonciations» annoncé dans le titre de l'ouvrage, et dont l'auteure approfondit les répercussions pour tout système traductionnel dans le chapitre suivant. Toujours à partir d'exemples concrets, le plus souvent - mais non exclusivement littéraires, l'auteure dresse un inventaire fort utile des lieux où se manifeste ce conflit des énonciations. À la fois nuancé et étendu, cet inventaire a un intérêt pédagogique certain, en ce qu'il réunit dans un seul cadre analytique cohérent un grand nombre de phénomènes, allant de «l'effet de traduction» (p. 130) aux «distorsions du rapport entre texte et hypersystème» (p. 168), en passant par la «sémantisation du nom propre par la traduction» (p. 142) et le décalage pragmatique (p. 152). Sur le plan théorique, cette systématisation des divers types d'indices de ré-énonciation sert de base à l'approfondissement, dans le chapitre suivant, de la question initiale de l'auteure sur les rapports entre la traduction et le discours rapporté. Plus précisément, il s'agit de décider «à quel type de discours rapporté - discours direct (comme dans le modele de Mossop), discours indirect, discours indirect libre, système hybride ou système sui generis - serait éventuellement assimilable la traduction» (p. 215). Sont abordées alors diverses questions: les conditions d'acceptabilité du modẻle citationnel de la traduction, l'inadéquation des systèmes canoniques comme modèles de la traduction, la traduction-pratique, la traduction transitive, la traduction comme récupération de l'infra-discursif, la traduction littérale de Berman.

Dans le dernier chapitre du livre, d'ordre synthétique, Barbara Folkart explore les répercussions de son modèle pour les discours traditionnels sur la traduction. Est mise en cause surtout la notion de fidélité: «Ainsi 
vouée au culte de la fidélité, la traduction se fait sacerdoce: le traducteur devient celui qui fait que soit dit en français comme en anglais le vouloirdire Originaire.» (p. 308) Car non seulement une telle conception du faire traducteur «érige en absolu ce qui en fait relève du contingent» (p. 308), mais elle repose aussi sur des assises idéologiques qui nient le travail du sujet traduisant, en valorisant même la ré-énonciation comme non-travail. Revenant en partie sur le chemin déjà déblayé, c'est-à-dire sur l'inévitable caractère transformateur de l'opération traductionnelle, l'auteure examine les divers obstacles ou contingences auxquels peut se heurter le sujet traduisant, au niveau cette fois-ci, non pas du faire ré-énonciatif, mais du faire interprétatif. Elle termine son ouvrage en abordant brièvement les rapports entre la traduction et l'altérité, et d'autres questions touchant à ce que l'on pourrait appeler l'épistémologie de la traduction. On trouve à la fin du livre un glossaire qui, malgré ses lacunes, en faciliterait l'utilisation pédagogique.

Il est impossible de rendre compte avec justesse d'un ouvrage de l'envergure de celui-ci. Si on a parfois l'impression d'une certaine surcharge terminologique, d'un cumul excessif de métaphores descriptives, cet ouvrage impressionne le plus souvent par son érudition et surtout par son attention aux détails réels du travail de traduction. $\AA$ la réflexion théorique se joint ainsi l'analyse nuancé d'exemples concrets, dans un mouvement constant de va-et-vient entre théorie et pratique qui contribue beaucoup aux résonances du travail de Barbara Folkart. Car il s'agit d'un ouvrage qui ne manquera pas de susciter de nombreux prolongements.

Agnès Whitfleld Université York 